\title{
A detailed study on the reflection component for the black hole candidate MAXI J1836-194
}

\author{
Yanting Dong, ${ }^{1,2 \star ~ J a v i e r ~ A . ~ G a r c i ́ a, ~}{ }^{3,4}$ Zhu Liu ${ }^{\odot},{ }^{1}$ Xueshan Zhao, ${ }^{1,2}$ \\ Xueying Zheng ${ }^{\odot 1,2}$ and Lijun Gou ${ }^{1,2 \star}$ \\ ${ }^{1}$ The National Astronomical Observatories, Chinese Academy of Sciences, Beijing 100101, China \\ ${ }^{2}$ University of Chinese Academy of Sciences, No.19(A) Yuquan Road, Shijingshan District, Beijing 100049, China \\ ${ }^{3}$ Cahill Centre for Astronomy and Astrophysics, California Institute of Technology, Pasadena, CA 91125, USA \\ ${ }^{4}$ Dr. Karl Remeis-Observatory and Erlangen Centre for Astroparticle Physics, Sternwartstr 7, D-96049 Bamberg, Germany
}

Accepted 2020 February 6. Received 2020 February 6; in original form 2019 December 15

\begin{abstract}
We present a detailed spectral analysis of the black hole candidate MAXI J1836-194. The source was caught in the intermediate state during its 2011 outburst by Suzaku and RXTE. We jointly fit the X-ray data from these two missions using the relxill model to study the reflection component, and a steep inner emissivity profile indicating a compact corona as the primary source is required in order to achieve a good fit. In addition, a reflection model with a lamp-post configuration ( $r e l x i l l l p$ ), which is normally invoked to explain the steep emissivity profile, gives a worse fit and is excluded at 99 per cent confidence level compared to relxill. We also explore the effect of the ionization gradient on the emissivity profile by fitting the data with two relativistic reflection components, and it is found that the inner emissivity flattens. These results may indicate that the ionization state of the disc is not constant. All the models above require a supersolar iron abundance higher than 4.5 . However, we find that the high-density version of reflionx can describe the same spectra even with solar iron abundance well. A moderate rotating black hole $\left(a_{*}=0.84-0.94\right)$ is consistently obtained by our models, which is in agreement with previously reported values.
\end{abstract}

Key words: accretion, accretion discs - black hole physics-relativistic processes-X-rays: individual: MAXI J1836-194.

\section{INTRODUCTION}

Galactic X-ray binaries are believed to be powered by accretion on to stellar-mass black holes or neutron stars. The gases in accretion disc emit thermal radiation in UV/X-ray band (Shakura \& Sunyaev 1973). Some fraction of the thermal photons from the disc are then inverse Compton scattered by energetic electrons in the hypothetically hot corona, producing a hard X-ray spectrum in the form of power law, i.e. $N(E) \propto E^{-\Gamma}$. A fraction of the highenergy photons will irradiate the cold accretion disc, generating the so-called X-ray reflection component (Fabian et al. 1989). The main features of the reflection spectrum are the fluorescent $\mathrm{Fe} \mathrm{K} \alpha$ emission line at energies of 6.4-6.97 keV (depend on the ionization state of the disc) and the Compton hump at 20-30 keV (Young, Ross \& Fabian 1999).

The profile of the reflection spectrum will be smeared due to the effects of Doppler shift, special relativity, and general relativity if it comes from the inner region of the accretion disc (Fabian

\footnotetext{
^E-mail: ytdong@nao.cas.cn (YD); 1gou@nao.cas.cn (LG)
}

et al. 2000). Observationally, the most prominent effect is that the intrinsically narrow $\mathrm{Fe} \mathrm{K} \alpha$ line is broadened and skewed to an asymmetric shape. The profile of the broad line, especially the red wing of the line, is directly linked to the inner radius of the accretion disc which is thought to be at the innermost stable circular orbit (ISCO), i.e. $R_{\text {in }}=R_{\mathrm{ISCO}}$. Thus, by modelling the broad iron line, we can deduce the spin of the black hole based on the relation between the spin and the ISCO (Bardeen, Press \& Teukolsky 1972). However, the line profile is readily affected by the subtraction of continuum and other components. Therefore, Reynolds (2014) pointed out that a more accurate measurement of the spin can be achieved by modelling the full reflection spectrum. The spin is one of the two key parameters to make a full description of a black hole. So far, we have measured dozens of stellar-mass black hole spins via X-ray reflection fitting method (Brenneman \& Reynolds 2006; Brenneman et al. 2011; Lohfink et al. 2012; Miller et al. 2013; Walton et al. 2013, 2019; García et al. 2015; Walton et al. 2016; Wang et al. 2017; García et al. 2018b; Tomsick et al. 2018; Xu et al. 2018; Tripathi et al. 2019).

The geometry of the corona, which is still unclear, will affect the profile of the reflection spectrum. Particularly, it has a significant 
Table 1. Details of the observations.

\begin{tabular}{lccccccc}
\hline Mission & Instrument & ObsID & MJD & $\begin{array}{c}\text { Start time } \\
\text { (in 2011) }\end{array}$ & $\begin{array}{c}\text { End time } \\
\text { (in 2011) }\end{array}$ & $\begin{array}{c}\text { Exp. }_{(\mathrm{s})} \\
\text { Count rate } \\
\left(\mathrm{cts} \mathrm{s}^{-1}\right)\end{array}$ \\
\hline Suzaku & XIS & 906003010 & 55818.43 & Sep 14, 10:12:23 & Sep 15, 10:50:14 & 19440 & 113.7 \\
& PIN & & & & & 35463 & 1.3 \\
RXTE & PCA & 96438010104 & 55818.84 & Sep 14, 20:12:00 & Sep 14, 20:31:44 & 1024 & 100.7 \\
& PCA & 96438010105 & 55819.16 & Sep 15, 03:56:32 & Sep 15, 05:52:48 & 4240 & 102.1 \\
\hline
\end{tabular}

${ }^{a}$ Count rate is measured in 1.2-10.0, 15.0-50.0, and 3.0-25.0 keV for XIS, PIN, and PCA, respectively.

impact on the emissivity profile of the reflection spectrum. The reflection emissivity profile is described by $\epsilon(r) \propto r^{-q}$, where $q$ is the emissivity index. It is normally assumed to be a broken power law with $q=q_{\text {in }}, r=R_{\mathrm{br}}$, and $q=q_{\text {out }}$, where $R_{\mathrm{br}}, q_{\mathrm{in}}$, and $q_{\text {out }}$ are the break radius, the emissivity index in the inner region, and in the outer region, respectively. In most cases, the break radius is usually hard to be constrained, and the emissivity index was only assumed to be a single value fixed at 3 (Novikov \& Thorne 1973; Shakura \& Sunyaev 1973; Reynolds \& Begelman 1997) due to limited photon statistics. Of course, a good constraint on the break radius and emissivity index is also obtained for a number of systems [both active galactic nuclei (AGNs) and binary systems], showing a steep inner index $\left(q_{\text {in }}>3\right)$ and small break radius $\left(R_{\mathrm{br}}<6 R_{\mathrm{g}}{ }^{1}\right)$, such as $1 \mathrm{H} 0419-577$ (Jiang et al. 2019), 1H0707-495 (Fabian \& Wilkins 2011; Fabian et al. 2012), IRAS 13224-3809 (Fabian et al. 2018), and Mrk 335 (Fabian et al. 2014; Wilkins \& Gallo 2015), as well as for black hole binaries, such as XTE J1752-223 (García et al. 2018b), Cyg X-1 (Wilkins et al. 2012), GRS 1915-105 (Miller et al. 2013), and MAXI J1535-571 (Xu et al. 2018). The steep emissivity profile is usually explained with an extremely compact corona locating close to the black hole, in which case a large fraction of the power-law emission will be focused towards the inner region as a result of lightbending effect (Miniutti \& Fabian 2004; Dauser et al. 2013). The $\mathrm{X}$-ray reflection emission profile has been successfully reproduced by lamp-post model (Duro et al. 2016; García et al. 2018b).

In addition, the disc ionization state can also affect the profile of the reflection emissivity, but it has not been discussed substantially in the previous studies. The ionization state of the disc at radius $r$ is defined as $\xi(r)=4 \pi F_{\mathrm{X}}(r) / n_{\mathrm{e}}(r)$, where $F_{\mathrm{X}}$ is the flux of the irradiation and $n_{\mathrm{e}}(r)$ is the electron density of the disc at radius $r$ (Fabian et al. 2000). As illustrated in Svoboda et al. (2012, see their fig. 3), the strong radius dependence of $F_{X}$ will naturally lead to the radial decrease of the disc ionization for any reasonable density profile of the disc. However, the ionization is always assumed to be constant in current reflection models. The simulations by Svoboda et al. (2012) and Kammoun et al. (2019) indicated that the ignorance of the ionization gradient will lead to an increase in the emissivity index.

In this work, we made a detailed study on the reflection spectrum of the stellar-mass black hole candidate MAXI J1836-194. The source was discovered as an X-ray transient by the MAXI/GSC (Negoro et al. 2011) and Swift/XRT (Kennea et al. 2011) on 2011 August 30. The coordinate of MAXI J1836-194 provided by Swift/XRT is RA/Dec. $(\mathrm{J} 2000)=278.93097 /-19.32004$. It was identified as a black hole candidate by studying its multiband properties (MillerJones et al. 2011; Nakahira et al. 2011; Rau, Greiner \& Sudilovsky 2011; Strohmayer \& Smith 2011). MAXI J1836-194 was active

\footnotetext{
${ }^{1} R_{\mathrm{g}}$ is the gravitational radius and is defined to be $R_{\mathrm{g}}=G M / \mathrm{c}^{2}$, where $G$ is the gravitational constant, $M$ is the mass of the black hole, and $c$ is the speed of light.
}

for about three months. However, it did not enter a soft state which suggested that this source experienced a failed outburst. López et al. (2019) inferred its companion as an M2 main-sequence star or later based on its near-infrared and optical properties. Low-frequency quasi-periodic oscillations are detected with Rossi X-ray Timing Explorer (RXTE) during the outburst (Jana et al. 2016).

MAXI J1836-194 was reported to have a spin parameter of $a_{*}=0.88 \pm 0.03$ at 90 percent confidence level by Reis et al. (2012), using one Suzaku spectrum during the intermediate state from its 2011 outburst. A relativistically broadened iron line was clearly shown. Reis et al. (2012) used a relativistic blurring model relconv to convolve with the reflection model refbhb (Ross \& Fabian 2007), in which the thermal emission of the disc and the reflection emission are included in a self-consistent way. They reported a steep broken power-law emissivity profile with $q_{\text {in }}>$ $7.3, q_{\text {out }}=3.19_{-0.05}^{+0.07}$, and $R_{\mathrm{br}}=3.6_{-0.1}^{+0.2} R_{\mathrm{g}}$.

In this paper, we re-analysed this Suzaku observation along with two simultaneous spectra taken by RXTE. A much more sophisticated reflection model, namely relxill (Dauser et al. 2014; García et al. 2014), is used. The model relxill is the combination of the ionized reflection produced by xillver (García \& Kallman 2010; García, Kallman \& Mushotzky 2011; García et al. 2013) and relativistic broadening based on relline (Dauser et al. 2010, 2013). The remarkable characteristic of this model is that the reflected flux can be calculated for each point on the disc, of which the light-bending effect is also taken into account. We explore the steep power-law index using the configurations with lamp-post and the ionization gradient, respectively, and also explore the effect of disc density on reflection spectrum.

The paper is organized as follows. We describe the observation and data reduction in Section 2, including both Suzaku and RXTE data. We present the detailed spectral analysis and results in Section 3. We make discussions and conclusions in Sections 4 and 5 , respectively.

\section{DATA SELECTION AND REDUCTION}

We searched the HEASARC data archive, and found one Suzaku and 74 RXTE observations in total. The Suzaku observation was carried out on 2011 September 14 (MJD 55818.43). The RXTE observations started on 2011 August 31 (MJD 55804.46) and ended on November 30 (MJD 55895.93). Ferrigno et al. (2012) and Jana et al. (2016) systematically studied MAXI J1836-194 using RXTE observations. In our work, only two RXTE observations (MJD 55818.84 and MJD 55819.16), which were simultaneously observed with Suzaku, were selected. The two spectra are in the intermediate state with the similar flux. Their hardness ratios, defined as the count rate at the energy band of $8.6-18 \mathrm{keV}$ to that at $5-8.6 \mathrm{keV}$, were calculated to be 0.65 (García et al. 2015). We listed the information including the ObsID, the start time, the end time, the exposure time, and the count rate of adopted observations in Table 1. 


\subsection{Suzaku observation}

There is one X-ray Imaging Spectrometer (XIS, Koyama et al. 2007) and one Hard X-ray detector (HXD, Takahashi et al. 2007) onboard Suzaku. The XIS consists of four CCDs. Since one of them, XIS2, had broken down in 2006 November, the remaining three, namely XIS0, XIS1, and XIS3, were operated in the ' $0.5 \mathrm{~s}$ burst mode'. The window size and editing mode were $1 / 4$ window and $3 \times 3 / 5 \times 5$, respectively. The HXD consisting of Si PIN photodiodes and GSO scintillation counters was operated in the 'normal' mode. The observation was performed at XIS nominal position. Following Reis et al. (2012), we only analysed the XIS0, XIS3, and PIN data. The archival data have been reprocessed and rescreened using the Suzaku pipeline (version 3.0.22.44) with the calibration data base hxd20110913, xis20160607, and xrt20110630. The latest calibration products of XIS on 2018 August 23 only improved the redistribution matrices around the $\mathrm{Si}-\mathrm{K}$ edge which will be ignored in our analysis. We generated the cleaned event files with HEASOFT version 6.19 following the Suzaku data analysis guide. ${ }^{2}$

For XIS, before extracting spectral products, we used the correction tools ${ }^{3}$ described in Yamada et al. (2012) to correct the mean position shift of the source, and to estimate the level of pile-up for XIS0 and XIS3. New attitude files were generated and were used as input for XISCOORD to create new cleaned event files. Unlike the pile-up estimation in Reis et al. (2012) in which they reported a maximum pile-up fraction of 2 per cent using the script PILE_EST ${ }^{4}$ (Davis 2001), we evaluated that the pile-up fraction of the source is larger than 3 per cent within the circle with a radius of 22.8 pixel ( 1 per cent at 58.7 pixel) from the source centre for XIS0 and larger than 3 per cent within the circle with a radius of 26.0 pixel ( 1 per cent at 60.6 pixel) for XIS3, respectively. The pile-up correction tool also created relevant region files. Yamada et al. (2012) recommended using the X-ray events from the region outside the radius with pileup fraction of 3 per cent or 1 per cent for spectral analysis. Here, we extracted spectra from an annular region that excluded events with pile-up fraction higher than 3 per cent.

The background spectra were extracted from a circular region with a radius of 100 arcsec, which is away from the source but still on the same chip. Then, we used XISRMFGEN and XISARFGEN to generate the new ancillary response files and redistribution matrix files for both XIS0 and XIS3, respectively. Finally, in order to increase the signal-to-noise ratio $(\mathrm{S} / \mathrm{N})$, we combined their spectra, backgrounds, and response files. We added systematic errors of 1 percent to the spectrum to take into account the calibration uncertainties.

For HXD, the spectrum (12-70 keV) observed by PIN diodes is used, while the spectrum (40-600 keV) detected by GSO scintillators is abandoned due to the low $\mathrm{S} / \mathrm{N}$. We obtained the appropriate response file (ae_hxd_pinxinome11_20110601.rsp) and the non-Xray background (NXB) file from the HXD team (Fukazawa et al. 2009). We extracted the background spectrum based on the NXB file and the total spectrum based on the cleaned events from the same good-time intervals. The 7 per cent dead time of the observed spectrum was corrected. The contribution from the cosmic Xray background (CXB), which contributes 5 percent of the PIN background, was estimated by simulation with 'fakeit' command in XSPEC, in which the model Boldt (1987) and the 'flat' response

\footnotetext{
${ }^{2}$ https://heasarc.gsfc.nasa.gov/docs/suzaku/analysis/abc/

${ }^{3}$ http://www-x.phys.se.tmu.ac.jp/syamada/ana/suzaku

${ }^{4}$ https://space.mit.edu/ASC/software/suzaku/pest.html
}

(ae_hxd_pinflate11_20110601.rsp) were used. Furthermore, the normalization of the model was adjusted so that the contribution from $\mathrm{CXB}$ is 5 percent. Then, the NXB and CXB were added together to obtain the total background spectrum.

For XIS data, due to the calibration issues below $\sim 1.0 \mathrm{keV}$, we only use the data in the $1.2-10.0 \mathrm{keV}$ energy range. The $1.6-2.0$ and 2.2-2.4 keV energy ranges are also excluded due to the $\mathrm{Si} \mathrm{K}$ and the Au M edge at $\sim 1.8$ and $\sim 2.2 \mathrm{keV}$, respectively. For HXD PIN data, we restrict its energy range between 15.0 and $50.0 \mathrm{keV}$. When both spectra are simultaneously fitted, a normalization factor of 1.16 is adopted as advised in the Suzaku data analysis guide.

\subsection{RXTE observations}

The standard RXTE products (the source, background, and response files) which were reduced from observations MJD 55818.84 and MJD 55819.16, were downloaded from the HEASARC data archive. The PCA spectra are used, while the HEXTE data are discarded due to the low S/N. The PCA were extracted from PCU2, the bestcalibrated detector. As a tradition, we added 0.6 per cent systematic uncertainties and rebinned the spectra with at least 25 photons within each bin. Following the spectral analysis of PCA spectra, we restrict our analysis in the energy range of 3.0-25.0 keV (Miller et al. 2009).

\section{SPECTRAL ANALYSIS AND RESULTS}

All spectra were analysed using XSPEC version 12.9.0g (Arnaud 1996). In order to model the Galactic absorption, we used the TBabs (Wilms, Allen \& McCray 2000) model. The solar abundances from Wilms et al. (2000) and the photoelectric cross-sections from Verner et al. (1996) were adopted. We fixed the column density to $0.2 \times 10^{22} \mathrm{~cm}^{-2}$ (Kennea et al. 2011), which was given by fitting the Swift/XRT observation. All uncertainties calculated for specified parameters in this paper are at 90 per cent confidence level $\left(\Delta \chi^{2}=\right.$ 2.71 ), unless noted particularly.

\subsection{Preliminary Suzaku spectral analysis}

We performed preliminary spectral fits to Suzaku spectrum with a model consisting of a power-law component (powerlaw) and a multitemperature blackbody (diskbb, Mitsuda et al. 1984), in specific, TBabs (diskbb+powerlaw). The 4-7 keV energy band were excluded to avoid the contribution from the potential broad $\mathrm{Fe} \mathrm{K} \alpha$ line. The best-fitting model as well as the ratio of the model to data are shown in Fig. 1. It is clear from Fig. 1 that the model does not fit the data well with $\chi_{v}^{2}=1.444(2924.13 / 2025)$. A skewed broad iron line profile, iron absorption edge, and a Compton hump are shown in the residues which could be the signature of the disc reflection.

The best-fitting photon index of powerlaw is $2.22 \pm 0.01$ and the temperature of diskbb is $0.439 \pm 0.002 \mathrm{keV}$. The total 2.0 $20.0 \mathrm{keV}$ unabsorbed flux is $\sim 1.8 \times 10^{-9} \mathrm{erg} \mathrm{cm}^{-2} \mathrm{~s}^{-1}, 22$ per cent of which is attributed to the thermal emission. Reis et al. (2012) reported a photon index of $\sim 2$, which is slightly smaller than our result. The fraction of the thermal emission in the total unabsorbed flux $\left(\sim 1.5 \times 10^{-9} \mathrm{erg} \mathrm{cm}^{-2} \mathrm{~s}^{-1}\right)$ estimated in their paper is 26 per cent. Our steeper power law and higher flux may be attributed to the pile-up correction process. Given a distance of $10 \mathrm{kpc}$ and a mass of $8 \mathrm{M}_{\odot}$ (Russell et al. 2014b), we calculated the Eddington ratio $L / L_{\text {Edd }}$ to be 11 per cent, suggesting a geometrically thin and optically thick accretion disc (McClintock et al. 2006). Thus, it is 




Figure 1. Unfolded Suzaku spectra fitted by ignoring the 4-7 keV energy band, but including when plotted. In the top panel, the total, diskbb, and power law components are black, red, and green dotted lines, respectively. In the bottom panel, the curvature in the data-to-model ratio plot shows the clear signature of the disc reflection.

reasonable to assume that the inner radius of the disc is at the ISCO $\left(R_{\text {in }}=R_{\mathrm{ISCO}}\right)$.

We also tried two other models, cutoffpl and nthComp (Zdziarski, Johnson \& Magdziarz 1996; Życki, Done \& Smith 1999), to fit the data. The model cutoffpl is a phenomenological power-law model with e-fold at high energies. The model nthComp is a physically motivated thermal Comptonization model in which the thermal seed photons from the disc gain energies by interacting with electrons in the hightemperature corona. The laor (Laor 1991) and smedge (Ebisawa et al. 1994) models were added to account for the reflection feature found in the ratio plot (Fig. 1). These two models are TBabs*smedge (diskbb+cutoffpl+laor) (hereafter $\mathrm{M}_{\text {off }}$ ) and TBabs* smedge (diskbb+nthComp+laor) (hereafter $\mathbf{M}_{\text {nth }}$ ), respectively. The central energy of the broad iron line was constrained between 6.40 and $6.97 \mathrm{keV}$ for the laor model, including all the possible ionization states of iron. The edge could change from 7.0 to $9.0 \mathrm{keV}$ and the smearing width was fixed at $7 \mathrm{keV}$ for the smedge model.

The $\mathrm{M}_{\text {off }}$ and $\mathrm{M}_{\text {nth }}$ can fit the data equally well with reduced chi-square of 1.062 and 1.065 for the same degrees of freedom, respectively. The best-fitting thermal temperature, normalization, and photon index of the two models are in agreement with each other, with $k T_{\text {disc }}=0.433 \pm 0.002 \mathrm{keV}, N_{\text {disc }}=5842_{-77}^{+96}$, and $\Gamma=2.156 \pm 0.008$ in $\mathrm{M}_{\text {off }}$, and $k T_{\text {disc }}=0.433 \pm 0.002 \mathrm{keV}$, $N_{\text {disc }}=5842_{-104}^{+109}$, and $\Gamma=2.150 \pm 0.016$ in $\mathrm{M}_{\text {nth }}$. In both cases, the high-energy cut-off parameters are not constrained and only upper limits $(300 \mathrm{keV})$ can be given. An inner radius smaller than $\sim 2.9 R_{\mathrm{g}}$ in the laor model indicates that the reflection emission arises from the innermost region around a rapidly rotating black hole. Moreover, the normalization of the di skbb model could also provide a measurement of the inner radius of the accretion disc $\left(R_{\text {in }}=D_{10 \mathrm{kpc}}\left[N_{\text {disc }} / \cos (i)\right]^{1 / 2}\right.$, where $D_{10 \mathrm{kpc}}$ is the source distance in unit of $10 \mathrm{kpc}$ ). With the inclination angle of 4-15 deg (Russell et al. 2014a) and the source distance of 4-10 kpc (Russell et al. 2014b), our fits $\left(N_{\text {disc }}=5842_{-104}^{+109}\right)$ indicate that the inner disc extends to 2.58-6.44 $R_{\mathrm{g}}$, which is in agreement with the value obtained by laor model.

\subsection{Relativistic reflection models for Suzaku and RXTE spectra}

In order to measure the spin of the black hole in MAXI J1836-194, we replaced the laor, smedge, and power-law models with the relxill model to fit the reflection emission and the power-law continuum. In addition, to better constrain the reflection component, we extended the energy band to $15 \mathrm{keV}$ by fitting the Suzaku and $R X T E$ simultaneously.

A multiplicative constant is included to account for the differences in the flux calibration between the RXTE/PCA and the Suzaku/XIS. The inclination angle $i$ was bounded between 4 and 15 deg based on the optical measurements (Russell et al. 2014a). The power-law continuum is described with the exponential cutoff power-law. The inner radius $R_{\text {in }}$ is equal to $R_{\mathrm{ISCO}}$. The outer radius $R_{\text {out }}$ is fixed at its default value: $R_{\text {out }}=400 R_{\mathrm{g}}$. We noted that, as demonstrated in Section 3.1, changing it to a thermal Comptonization model will not affect our results. The high-energy cut-off $E_{\text {cut }}$ is equal to $\sim(2-3) k T_{\mathrm{e}}$, in which $k T_{\mathrm{e}}$ is the electron temperature of the corona. $E_{\text {cut }}$ is fixed at its default value of $300 \mathrm{keV}$.

The power-law continuum was assumed to come from an extended corona (Model 1). A broken power-law emissivity profile was adopted. The outer index was fixed at its canonical value $\left(q_{\text {out }}=\right.$ 3 ), while the inner index $q_{\text {in }}$ and the break radius $R_{\mathrm{br}}$ were set free. This model gives a much better fit with $\chi_{v}^{2}=1.002(2118.76 / 2115)$, compared to the initial fit with TBabs (diskbb+powerlaw) in Section 3.1. The best-fitting results for each parameter can be found in Table 2. The best-fitting model is shown in the top left panel in Fig. 2. The residuals with $1 \sigma$ of the best-fitting are shown in the top two panels in Fig. 3.

The temperature and the normalization of the thermal emission are $0.436_{-0.002}^{+0.003} \mathrm{keV}$ and $5660_{-168}^{+129}$, respectively. The photon index is $2.11_{-0.01}^{+0.02}$. The inner index of the emissivity profile is $6.42_{-1.83}^{+1.10}$ and the break radius is $4.45_{-0.64}^{+0.72} R_{\mathrm{g}}$, implying that the corona is compact and the flux of the reflected emission decreases dramatically within the break radius. The best-fitting spin parameter is at a moderate value of $0.88_{-0.04}^{+0.03}$. The fit gives an upper limit of $9 \mathrm{deg}$ for the inclination angle. The iron abundance $A_{\mathrm{Fe}}$ is $4.99_{-0.68}^{+1.02}$. The logarithmic ionization state is $3.67_{-0.12}^{+0.05}$. The reflection fraction which defines the photon fraction hitting the accretion disc (Dauser et al. 2014) is $0.45_{-0.06}^{+0.12}$. When the outer index is free, it is constrained to be $3.31_{-0.04}^{+0.05}$, and the inner index and the iron abundance only obtained their lower limit of 7.46 and 7.29, respectively. The values of the inner index and the iron abundance are too large to be considered as physical. In any event, the value of the spin parameter we cared most is not affected. Therefore, we will set the outer index at 3 in the rest of the fits for simplicity.

In previous studies, a narrow $\mathrm{Fe} \mathrm{K} \alpha$ line, which could be produced in the region far away from the central black hole, was already detected in the X-ray spectra of some black hole X-ray binaries, such as GX 339-4 (García et al. 2015) and Cyg X-1 (Tomsick et al. 2018). However, there is no clear evidence for such a narrow line in the X-ray spectrum of MAXI J1836-194 (see top two panels of Fig. 3). To further test the significance of this component, we added the xillver model, which is used to model unblurred reflection component, to Model 1. We found that the inclusion of this component did not significantly improve the fit with $\chi_{v}^{2}=1.002(2118.39 / 2114)$. The intensity of the relativistic 
Table 2. Best-fitting parameters with relativistic models.

\begin{tabular}{|c|c|c|c|c|}
\hline Parameter & Model 1 & Model 2 & Model 3 & Model 4 \\
\hline \multicolumn{5}{|c|}{ Suzaku } \\
\hline \multicolumn{5}{|c|}{ Multitemperature blackbody } \\
\hline$k T_{\text {disc }}(\mathrm{keV})$ & $0.436_{-0.002}^{+0.003}$ & $0.4380(0.0004)^{\mathrm{a}}$ & $0.434(0.003)$ & $0.412_{-0.002}^{+0.010}$ \\
\hline$N_{\text {disc }}$ & $5660_{-168}^{+129}$ & $5588(18)$ & $5887_{-191}^{+164}$ & $6484_{-715}^{+477}$ \\
\hline \multicolumn{5}{|c|}{ Power-law continuum plus relativistic reflection } \\
\hline$\Gamma$ & $2.11_{-0.01}^{+0.02}$ & $2.109(0.002)$ & $2.00_{-0.01}^{+0.06}$ & $2.02_{-0.02}^{+0.01}$ \\
\hline$h\left(R_{\mathrm{g}}\right)$ & - & $2.36_{-0.17}^{+0.53}$ & - & - \\
\hline$q_{\text {in }}$ & $6.42_{-1.83}^{+1.10}$ & - & $4.38_{-0.59}^{+0.57}$ & $>5.95$ \\
\hline$R_{\mathrm{br}}\left(R_{\mathrm{g}}\right)$ & $4.45_{-0.64}^{+0.72}$ & - & $5.34_{-0.76}^{+0.72}$ & $4.01_{-0.29}^{+0.36}$ \\
\hline$\underset{i(\operatorname{deg})^{b}}{a_{*}}$ & $\begin{array}{c}0.88_{-0.04}^{+0.03} \\
5^{+4}\end{array}$ & $\begin{array}{c}0.934(0.005) \\
15_{-1}\end{array}$ & $\begin{array}{c}0.91_{-0.04}^{+0.03} \\
4^{+3}\end{array}$ & $\begin{array}{c}0.88_{-0.03}^{+0.02} \\
5^{+3}\end{array}$ \\
\hline$A_{\mathrm{Fe}}$ & $4.99_{-0.68}^{+1.02}$ & $>9.35$ & $>6.62$ & $1(\mathrm{f})^{c}$ \\
\hline $\log \xi_{1}$ & $3.67_{-0.12}^{+0.05}$ & $3.63(0.05)$ & $4.23_{-0.13}^{+0.12}$ & $2.50_{-0.09}^{+0.11}$ \\
\hline$R_{\text {ref }}$ & $0.45_{-0.06}^{+0.12}$ & $1.33(0.02)$ & $0.65_{-0.25}^{+0.45}$ & - \\
\hline $\log \xi_{2}$ & - & - & $3.47_{-0.06}^{+0.10}$ & - \\
\hline $\log n_{e}$ & - & - & - & $>21.83$ \\
\hline$N_{\text {cutoffpl }}$ & - & - & - & $0.25(0.01)$ \\
\hline$N_{\text {relxill }}\left(\times 10^{-2}\right)$ & $0.38_{-0.04}^{+0.03}$ & $6.24_{-0.01}^{+1.96}$ & $0.21_{-0.06}^{+0.08}$ & - \\
\hline$N_{\text {relxill }_{2}}\left(\times 10^{-2}\right)$ & - & - & $0.119_{-0.02}^{+0.08}$ & - \\
\hline$N_{\text {reflionx_hd }}$ & - & - & - & $1.17_{-0.10}^{+0.38}$ \\
\hline
\end{tabular}

RXTE

Cross-Normalization constant (relative to Suzaku/XIS)

$$
\mathrm{C}
$$$$
1.035_{-0.005}^{+0.004}
$$

$1.035(0.004)$

$\chi^{2} / v$

$2118.76 / 2115$

1.002

$2144.58 / 2116$

1.014
$1.034(0.005)$

$1.035(0.005)$

$2107.01 / 2113 \quad 2093.68 / 2115$

0.997 reflection is also 30 times stronger than it. Additionally, it did not affect the values of other parameters, such as the spin, the inclination angle, and the iron abundance. Therefore, we did not include this distant reflection in our fits.

As to the geometry of the corona, it is still unclear, but there are two popular models: extended geometry (Wilkins \& Fabian 2012) and lamp-post configuration (Matt, Perola \& Piro 1991; Martocchia \& Matt 1996). In Model 1, we have assumed a broken power-law emissivity to explore the extended corona, in which case we found that a compact corona is required. In order to test the effect of different geometry on the spin, we also tried to fit the data with a lamp-post configuration using the model relxilllp (Model 2). The lamp-post model leads to a slightly worse fit with $\chi_{\nu}^{2}=1.014$ (2144.58/2116) compared to Model 1 . The best-fitting results of
Model 2 are shown in Table 2. The model components are shown in the top right panel in Fig. 2 and the residuals with $1 \sigma$ are shown in the second top two panels in Fig. 3.

The fitted value for the parameters in Model 2 are listed here. The temperature and the normalization of the thermal disc are $0.4380 \pm 0.0004 \mathrm{keV}$ and $5588 \pm 18$, respectively. The photon index is $2.109 \pm 0.002$. The logarithmic ionization state is $3.63 \pm 0.05$. These best-fitting parameters are consistent with the results found in Model 1. The height of the point source is $2.36_{-0.17}^{+0.53} R_{\mathrm{g}}$, which indicates that a compact corona is located closely to the black hole. The spin parameter of the black hole is $0.934 \pm 0.005$, slightly higher than that in Model 1. The inclination angle is larger than $14 \mathrm{deg}$ but pegged at the upper limit of $15 \mathrm{deg}$. However, the inclination angle was constrained to be $\sim 16-19$ deg when 

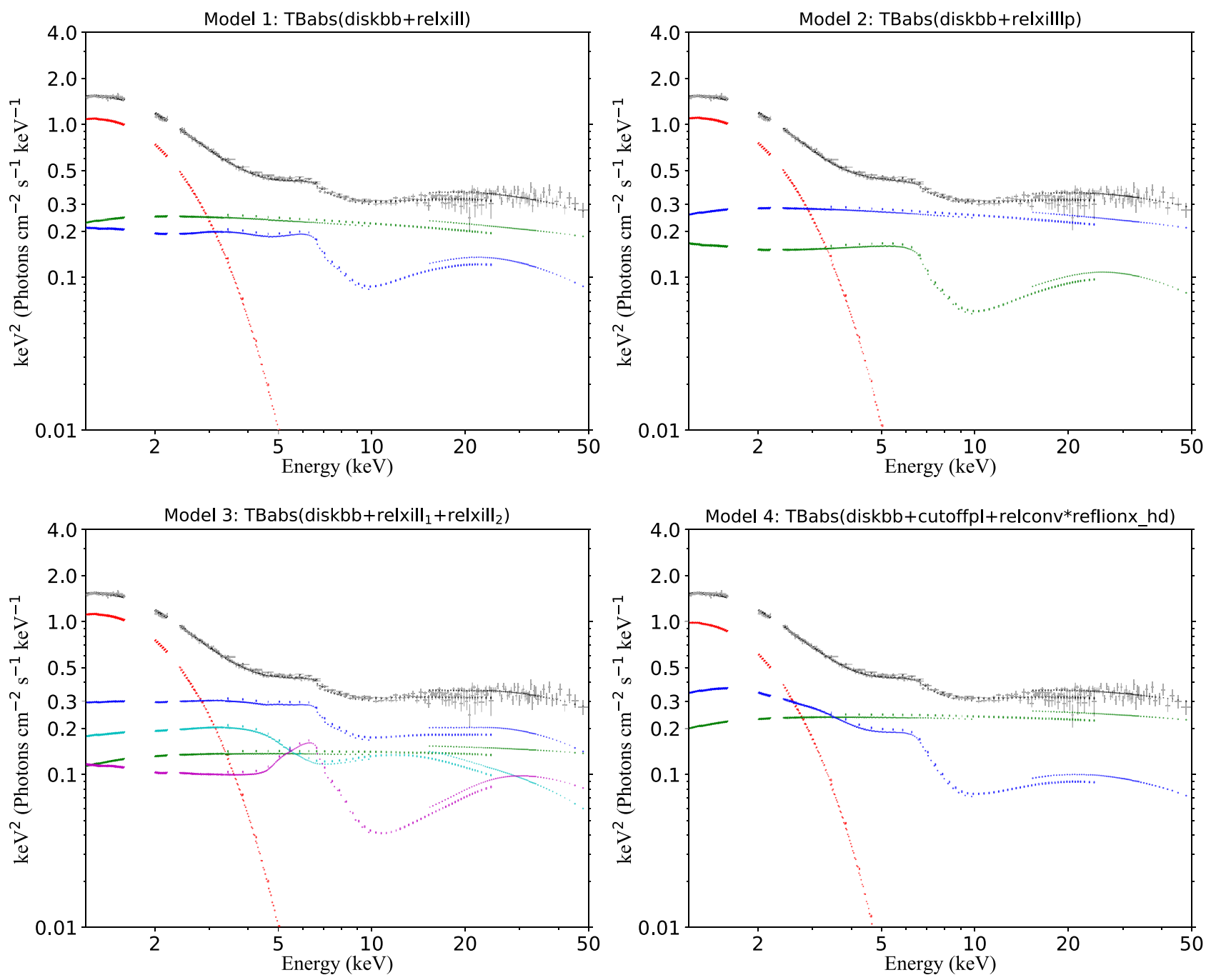

Figure 2. Unfolded Suzaku and RXTE spectra fitted with four models. In all panels, the total, the thermal, the power-law, and the reflection components are black, red, green, and blue dotted lines, respectively. In the bottom left panel, we use cyan and purple dotted lines to describe the relativistic reflection component in the inner disc and the outer disc, respectively, and blue to describe the sum of the two reflection components.

the upper bound is set at a much larger value, which is still consistent with a low disc inclination angle. The iron abundance $A_{\mathrm{Fe}}$ is larger than 9.35. Moreover, Model 2 requires a stronger illumination of $N_{\text {relxill }}=\left(6.24_{-0.01}^{+1.96}\right) \times 10^{-2}$ and reflection fraction of $R_{\text {ref }}=1.33 \pm 0.02$ compared to those in Model 1 .

It is suggested that the constant ionization in current models may lead to a steep emissivity index. To explore the effect of constant ionization on steep emissivity index, we fit the data with a dual-relativistic reflection model, i.e. a second relativistic reflection component was added in Model 1 (García et al. 2019). This new model is defined as Model 3. We use R1 and R2 to differentiate the two relxill models. In $\mathrm{R} 1$, the outer radius $R_{\text {out }}$ is equal to the break radius $R_{\mathrm{br}}$, while in $\mathrm{R} 2$ the inner radius $R_{\mathrm{in}}$ is linked to $R_{\mathrm{out}}$ in $\mathrm{R} 1$. The other parameters, $q_{\mathrm{in}}, q_{\mathrm{out}}$, and $R_{\mathrm{br}}$ in R2, are fixed at their default values, i.e. $q_{\mathrm{in}}=3, q_{\mathrm{out}}=3$, and $R_{\mathrm{br}}=15 R_{\mathrm{g}}$. The ionization parameters in R1 and R2 are free. The normalization parameter in $\mathrm{R} 2$ is also free. The remaining parameters in $\mathrm{R} 1$ and $\mathrm{R} 2$ are linked together.

Compared with Model 1, Model 3 provides a better statistics with $\chi_{v}^{2}=0.997(2107.01 / 2113)$. The best-fitting parameters of Model
3 are presented in Table 2. The model components are shown in the bottom left panel in Fig. 2 and the residuals with $1 \sigma$ of the bestfitting are shown in the third top two panels in Fig. 3. For Model 3 , the temperature and the normalization of the thermal disc are $0.434 \pm 0.003 \mathrm{keV}$ and $5887_{-191}^{+164}$, respectively. The photon index is $2.00_{-0.01}^{+0.06}$. The spin parameter of the black hole is $0.91_{-0.04}^{+0.03}$. The inclination angle is smaller than $7 \mathrm{deg}$. The iron abundance $A_{\mathrm{Fe}}$ is larger than 6.62 . These parameters are consistent with the bestfitting parameters in Model 1 . The best-fitting emissivity index of $\mathrm{R} 1$ is $4.38_{-0.59}^{+0.57}$ with the break radius of $5.34_{-0.76}^{+0.72} R_{g}$, which indicate a more extended corona than that shown in Model 1. The logarithmic ionization state of R1 and R2 are $4.23_{-0.13}^{+0.12}$ and $3.47_{-0.06}^{+0.10}$, respectively, which may indicate ionization gradient on the surface of the disc.

We noticed that the iron abundances in all three models prefer supersolar. Forcing $A_{\mathrm{Fe}}$ at the solar abundance in Model 1 will lead to a worse fit ( $\Delta \chi^{2} \sim 156.63$ with one degree of freedom), but the spin parameter $a_{*}$ is $0.79_{-0.10}^{+0.06}$, which still indicates a moderate rotating black hole. We also tried a new version of reflionx model (reflionx_hd) with the electron density as a free parameter 

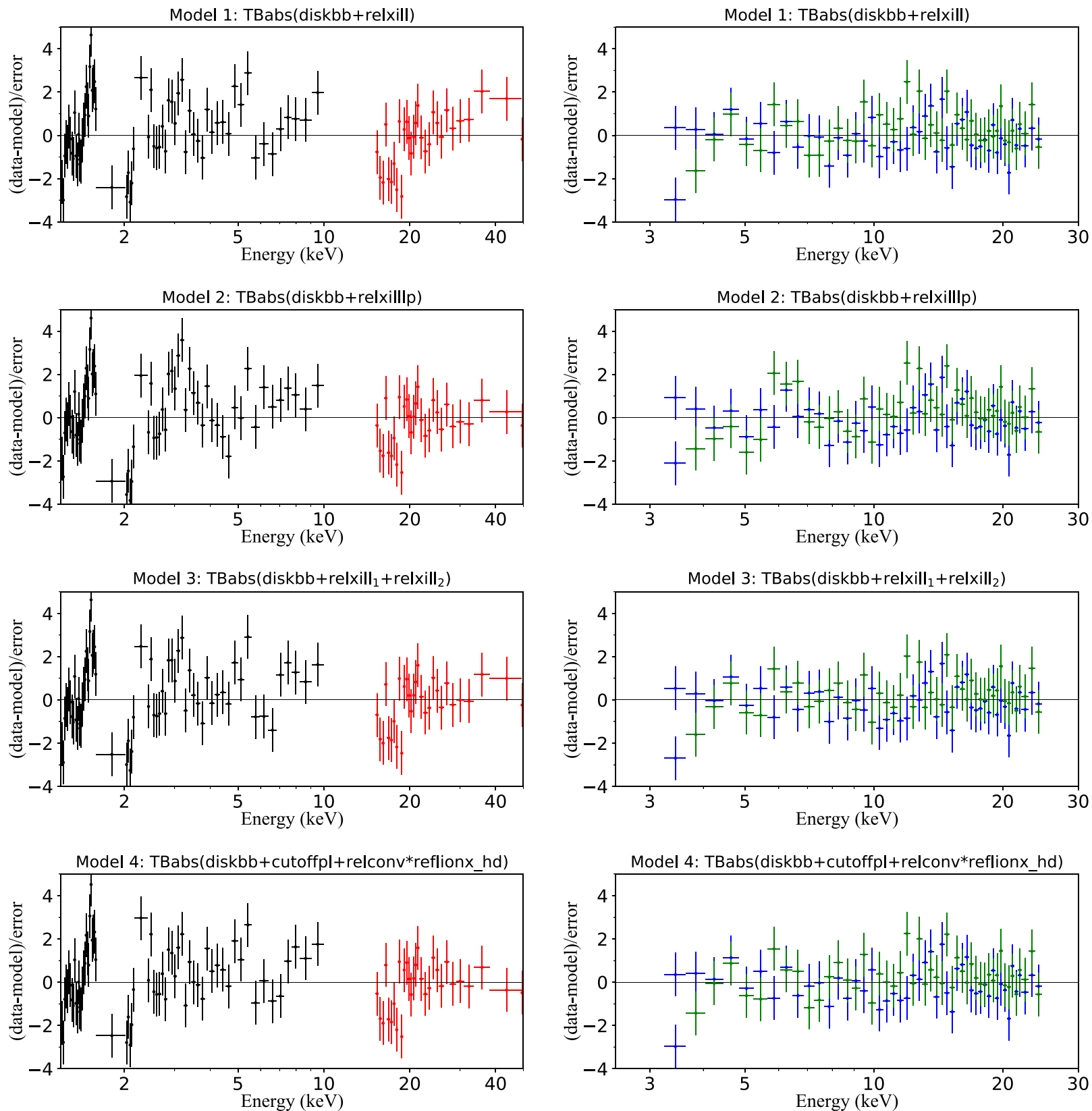

Figure 3. The residuals with $1 \sigma$ for four models are shown in plot. The black and red represent the result of fit to Suzaku/XIS and PIN spectra, respectively (left-hand panels). The green and blue represent the result of fit to RXTE/PCA spectra obtained in MJD 55818.84 and 55819.16, respectively (right-hand panels). These spectra were fitted together, but are shown separately for clarity. The XIS and HXD spectra are binned in XSPEC only for visual clarity.

(Tomsick et al. 2018). The iron abundance is fixed at solar abundance. The rel conv is used to account for the relativistic effect on the reflection emission, while the cutoffpl is adopted to model the power-law emission. This model is defined as Model 4. Model 4 only improve the fit slightly with $\chi_{v}^{2}=0.990(2093.68 / 2115)$. The best-fitting parameters are presented in Table 2 . The model components are shown in the bottom right panel in Fig. 2 and the residuals with $1 \sigma$ of the best-fitting are shown in the bottom two panels in Fig. 3. It does not show any significant residual either in Suzaku or in RXTE data. The spin parameter of the black hole is $0.88_{-0.03}^{+0.02}$. The best-fitting inclination angle is smaller than $8 \mathrm{deg}$. The logarithmic ionization parameter is $2.50_{-0.09}^{+0.11}$, which is smaller than that found in other models. The most important thing is that the density is larger than $10^{21} \mathrm{~cm}^{-3}$, which is significantly larger than the assumed disc density of $10^{15} \mathrm{~cm}^{-3}$ in models relxill and relxillip.

\section{DISCUSSION}

In this paper, we analysed the broad-band X-ray data of MAXI J1836-194 in the intermediate state, using the simultaneously observed X-ray data from Suzaku and RXTE. The unabsorbed Eddington ratio is approximately 11 per cent in which the accretion disc is geometrically thin and optically thick, suggesting that the inner edge of the disc has already reached the ISCO radius. The sophisticated relativistic reflection models (relxill(lp) and 
reflionx_hd) are used to fit the data and measure the spin of the black hole.

We fitted the data with four models. In Model 1, we assumed an extended corona covering above the accretion disc with a broken power-law emissivity. In Model 2, we assumed a lamp-post configuration in which a point-like corona is located above the black hole. In Model 3, to explore the effect of ionization gradient, we fitted the data with a model consisting of two relativistic reflection components. One of the two components is used to fit the reflection from the inner region of the accretion disc, while the other one is used to account for the radiation from the outer region. In Model 4, we applied a new version of reflionx with the electron density to be a free parameter. As can be seen from Fig. 3, all the four models can fit the data well, giving acceptable fits.

According to best-fitting results shown in Table 2, a thermal component with the temperature of approximately $0.4 \mathrm{keV}$ is required in all models. But the thermal emission below $5 \mathrm{keV}$ in the Model 4 is slightly smaller than that in other three models (see Fig. 2). The fact that the index of the powerlaw component is $\sim 2$, together with the disc fraction of 26 per cent, implies that the source is in the intermediate state. However, the power-law index is slightly flatter in Model 3-4. For Model 3, this may be due to the more contribution from thermal emission, while for Model 4, it is led by the more contribution from the reflection (see Fig. 2) at soft $\mathrm{X}$-ray band. The inclination angle of the disc is very low, which is consistent with the optical measurement made by VLT (Russell et al. 2014a).

\subsection{Steep inner emissivity index}

A steep inner emissivity index $\left(6.42_{-1.83}^{+1.10}\right)$ and a small break radius $\left(4.45_{-0.64}^{+0.72} R_{\mathrm{g}}\right)$, which is common in X-ray binary and AGN, is found in Model 1 (assuming the broken power-law emissivity). We showed the contour plot for the spin and the emissivity index for Model 1 in the right-hand panel in Fig. 4. A mildly inverse relationship between these two parameters is shown. Steep emissivity indexes $\left(q_{\text {in }}>4\right)$ are required for any value in the narrow spin range. Fixing $q_{\text {in }}$ at 3 and $R_{\mathrm{br}}$ at $15 R_{\mathrm{g}}$ will lead to a worse fit with $\chi_{v}^{2}=1.033$ (2186.91/2117). The spin parameter $a_{*}$ is inferred to be larger than 0.93 .

The steep inner index may be explained with a compact corona close to the black hole. Our Model 2 (assuming a point-like corona) is used to test this explanation. As what we expected, a very low height ( $h=2.36_{-0.17}^{+0.53} R_{\mathrm{g}}$ ) of the compact corona is found. The lightbending effect focuses more photons on the inner region of the disc, which steepens the inner index. This effect may also lead the reflection fraction parameter larger than unity. In our case, the reflection fraction $R_{\text {ref }}$ is $1.33 \pm 0.02$. However, we found that the reflection component is smaller than that in Model 1 (compare the top two panels in Fig. 2), and the reflection component is weaker than the power-law component. We think it is because, in this configuration, the spin parameter $a_{*} \sim 0.93$ corresponds to the $R_{\text {ISCO }}$ of $2.10 R_{\mathrm{g}}$, then some of the power-law photons that should have hit the accretion disc fall into the gap between the black hole and the disc or directly into the black hole. In addition, we found that the iron absorption edge is larger than that in Model 1 (Fig. 2). The steeper iron edge is due to the suddenly increasing iron abundance $\left(A_{\mathrm{Fe}} \sim 10\right)$, which is an extremely unphysical value. Moreover, Model 2 is excluded at 99 per cent confidence level compared to the Model 1. We will then disregard Model 2.

The steep emissivity profile might be caused by the simple assumption of a constant ionization along the radius in the reflection model. The dual-relativistic reflection model (Model 3) found two ionization states $\left(\log \xi_{1}=4.23_{-0.13}^{+0.12}\right.$ and $\left.\log \xi_{2}=3.47_{-0.06}^{+0.10}\right)$ for different region of the disc. Model 3 is favoured over Model 1 as suggested from the F-test (with a p-value of 0.003). Model 3 gives an inner emissivity index of $4.38_{-0.59}^{+0.57}$ and a break radius of $5.34_{-0.76}^{+0.72} R_{g}$ which indicate a more extended corona than that found in Model 1. Our results give a tentative evidence for ionization gradient along the radius of the disc. According to the bottom left panel in Fig. 2, we obtain different reflection profiles of the relxill model for different ionization parameters. The strength of the two reflection components is comparable. The total reflection is stronger than that in other models. The softer and more ionized reflection component comes from the innermost region. As suggested in Svoboda et al. (2012), when a constant ionization is assumed in the reflection model, the flux from the innermost region may be significantly underestimated, which then leads to a steep emissivity index. The emissivity found in Model 1 is indeed steeper than that in Model 3, consistent with the prediction from the simulation.

\subsection{High iron abundance}

Model 1 and Model 3 require an extremely supersolar iron abundance. We showed the contour plot for the spin and the iron abundance for Model 1 in the left-hand panel in Fig. 4. The plot suggests a slightly positive correlation between the two parameters. Moreover, the iron abundance is obtained to be 3.5-8 and the spin parameter is well constrained to be $0.81-0.94$ at 99 percent confidence. García et al. (2018a) pointed out the most likely explanation for the supersolar iron abundance is the lack of models with very high density. The new version of reflionx with the electron density free allows the $A_{\mathrm{Fe}}$ parameter to return back to the solar abundance. Moreover, it provides the best fit among our four models. The high disc density $\left(n_{\mathrm{e}}>10^{21} \mathrm{~cm}^{-3}\right)$ is suggested by the model. A significantly soft excess bellow $2 \mathrm{keV}$ is found in the bottom right panel in Fig. 2. García et al. (2016) demonstrated that the increasing free-free absorption heats the gas, which results in an increase of the gas temperature when density is larger than $10^{17}$ $\mathrm{cm}^{-3}$. Then, it will increase the flux at low energies $(<2 \mathrm{keV})$. This effect has been shown in Cyg X-1 (Tomsick et al. 2018). We noticed that the ionization state decreases, which follows its relationship with density (see the formula in Section 1). It is noted that the high density does not affect our spin measurement.

\subsection{Spin constraints}

The spin parameter can be constrained with the best-fitting value $a_{*}=0.88_{-0.04}^{+0.03}$ for Model 1 . However, the steep inner emissivity index $\left(q_{\text {in }}=6.42_{-1.83}^{+1.10}\right)$ and the high iron abundance $\left(A_{\mathrm{Fe}}=4.99_{-0.68}^{+1.02}\right)$ are also given by Model 1, which calls into question the reliability of the results. When the ionization gradient is considered in Model 3 , the inner emissivity index flattens $\left(q_{\text {in }}=4.38_{-0.59}^{+0.57}\right)$, and the spin parameter changes to $0.91_{-0.04}^{+0.03}$. We also used the high-density model to fit the data in Model 4. It is found that the iron abundance can be reduced to the solar abundance, and the spin parameter is $a_{*}=0.88_{-0.03}^{+0.02}$. The spins measured by different models consistently indicate a moderate rotating black hole (see Fig. 5) in MAXI J1836-194.

Our new spin result is in agreement with that shown in Reis et al. (2012). They used model refbhb together with relativistic smearing model to account for the physical processes happening in MAXI J1836-194 system. The refbhb model combines the thermal disc and reflection emission in a consistent way, which 

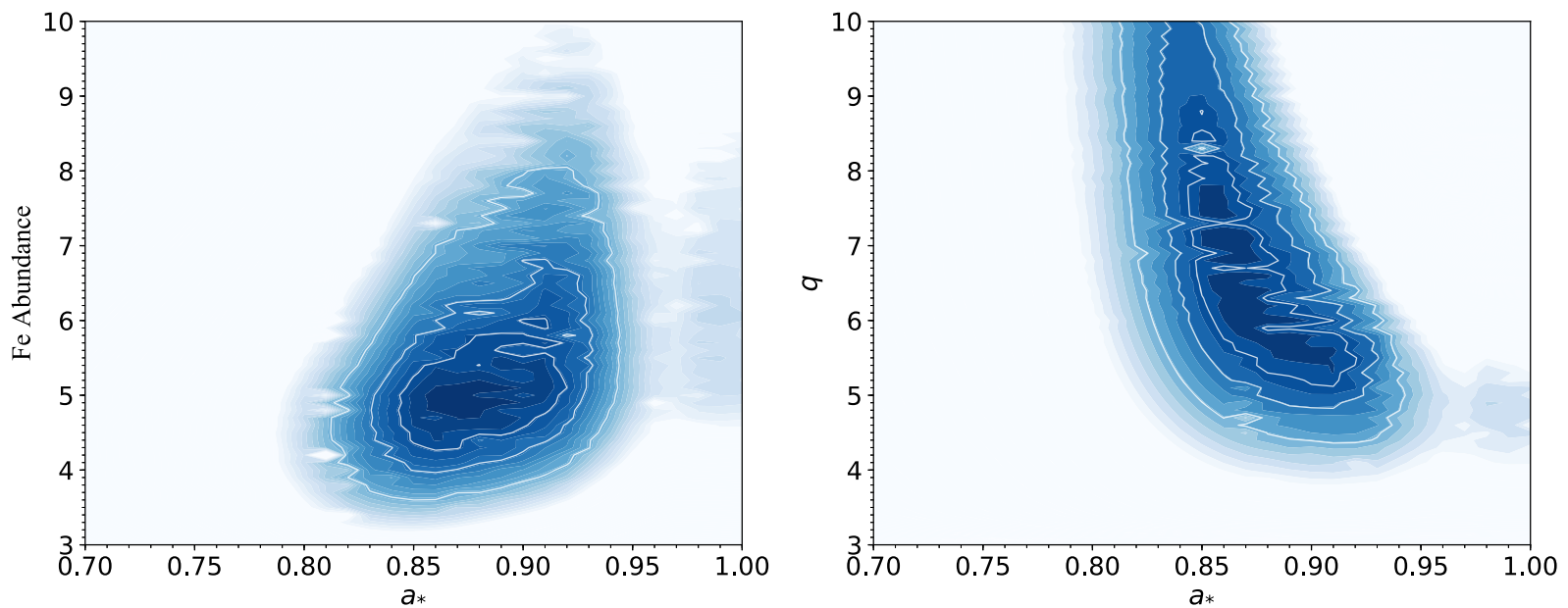

Figure 4. The correlation between the spin parameter $a_{*}$ and the iron abundance $A_{\mathrm{Fe}}$ and the correlation between the spin parameter $a_{*}$ and the inner index $q_{\mathrm{in}}$ of emissivity are calculated with the command 'steppar' based on Model 1. For visual guide, probability contours of 68 per cent, 90 per cent, and 99 per cent are shown in white solid lines.

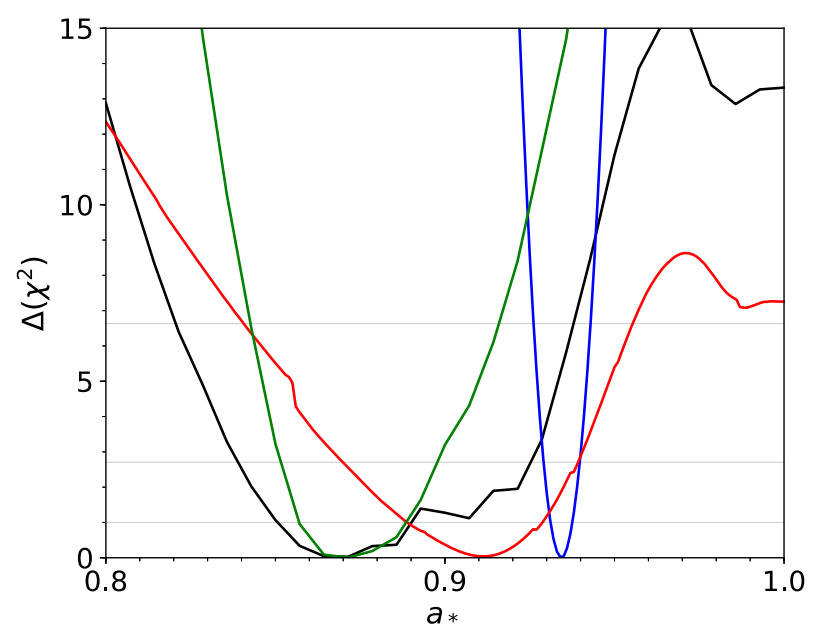

Figure 5. In XSPEC, using 'steppar' command to search the best fit for 20 values of $a_{*}$ from 0.8 to 1 for Model $1-4$. The black, blue, red, and green solid lines are used to represent the results obtained from Model 1, 2, 3, and 4 successively. The 68 per cent, 90 per cent, and 99 per cent confidence intervals are indicated by the grey lines.

works well when the disc temperature is so high that the Compton broadening of $\mathrm{Fe}$ line is of significance. But in our case, the fraction of the thermal emission to the total unabsorbed flux in the $2.0-20.0 \mathrm{keV}$ band is only 22 percent, meaning the thermal emission from the disc is not dominant in the total emission in the observation we work on. The physical model without including the thermal emission we use therefore could work well. In the meantime, our model (relxill) convolves the reflection with relativistic effect, which is a more advanced method to calculate the emission near the black hole, but refbhb needs to be calculated externally.

\section{CONCLUSIONS}

In this paper, to measure the spin of the black hole candidate in binary system MAXI J1836-194 well, we first evaluated and eliminated pile-up effect for Suzaku observation, using the newest pipeline provided by Yamada et al. (2012), and we also included two simultaneous observations by RXTE to increase the spectral energy band. We fit the spectra jointly with the sophisticated relativistic reflection models and a moderate rotating black hole is obtained. The spin parameter is well estimated to be within 0.84-0.94, which agrees well with the spin obtained by Reis et al. (2012). The inclination angle is also constrained, which is consistent with the one obtained by the optical measurement. In the previous fit, there are two potential problems, one is the steep emissivity index, and the other is supersolar abundance. We also tried to solve them. As to the steep emissivity index, it can be solved by introducing an ionization gradient. The supersolar iron abundance can be relieved by increasing the electron density. Of course, the high-density model with solar iron abundance also gives a similar spin with the models which assume the constant density. We hope the future reflection model can incorporate the ionization gradient and allow the higher density to study the spin of black hole.

\section{ACKNOWLEDGEMENTS}

We thank the useful discussions with Prof. S. Yamada on extracting Suzaku spectrum. We thank the high-density model provided by Dr. John A. Tomsick. We thank the valuable discussions with Dr. Erlin Qiao. We also thank the reviewer for her valuable comments. Lijun Gou is supported by the National Program on Key Research and Development Project through grant No. 2016YFA0400804, and by the National Natural Science Foundation of China with grant No. U1838114, and by the Strategic Priority Research Program of the Chinese Academy of Sciences through grant No. XDB23040100. This research has made use of data obtained from the Suzaku satellite, a collaborative mission between the space agencies of Japan (JAXA) and the USA (NASA), and also has made use of standard data products obtained from the RXTE satellite and the RXTE Guest Observer Facility (GOF). This research has made use of data and/or software provided by the High Energy Astrophysics Science Archive Research Centre (HEASARC), which is a service of the Astrophysics Science Division at NASA/GSFC and the High Energy Astrophysics Division of the Smithsonian Astrophysical Observatory. 


\section{REFERENCES}

Arnaud K. A., 1996, in Jacoby G. H., Barnes J., eds, ASP Conf. Ser. Vol. 101, Astronomical Data Analysis Software and Systems V. Astron. Soc. Pac., San Francisco, p. 17

Bardeen J. M., Press W. H., Teukolsky S. A., 1972, ApJ, 178, 347

Boldt E., 1987, in Hewitt A., Burbidge G., Fang L. Z., eds, Proc. IAU Symp. Vol. 124, Observational Cosmology. Kluwer, Dordrecht, p. 611

Brenneman L. W., Reynolds C. S., 2006, ApJ, 652, 1028

Brenneman L. W. et al., 2011, ApJ, 736, 103

Dauser T., Garcia J., Wilms J., Böck M., Brenneman L. W., Falanga M., Fukumura K., Reynolds C. S., 2013, MNRAS, 430, 1694

Dauser T., García J., Parker M. L., Fabian A. C., Wilms J., 2014, MNRAS, 444, L100

Dauser T., Wilms J., Reynolds C. S., Brenneman L. W., 2010, MNRAS, 409, 1534

Davis J. E., 2001, ApJ, 562, 575

Duro R. et al., 2016, A\&A, 589, A14

Ebisawa K. et al., 1994, PASJ, 46, 375

Fabian A. C., Iwasawa K., Reynolds C. S., Young A. J., 2000, PASP, 112, 1145

Fabian A. C., Rees M. J., Stella L., White N. E., 1989, MNRAS, 238, 729

Fabian A. C., Wilkins D. R., 2011, MNRAS, 414, 1269

Fabian A. C. et al., 2012, MNRAS, 419, 116

Fabian A. C. et al., 2014, MNRAS, 443, 1723

Fabian A. C. et al., 2018, MNRAS, 477, 3711

Ferrigno C., Bozzo E., Del Santo M., Capitanio F., 2012, A\&A, 537, L7

Fukazawa Y. et al., 2009, PASJ, 61, S17

García J., Dauser T., Reynolds C. S., Kallman T. R., McClintock J. E., Wilms J., Eikmann W., 2013, ApJ, 768, 146

García J., Kallman T. R., 2010, ApJ, 718, 695

García J., Kallman T. R., Mushotzky R. F., 2011, ApJ, 731, 131

García J. A., Fabian A. C., Kallman T. R., Dauser T., Parker M. L., McClintock J. E., Steiner J. F., Wilms J., 2016, MNRAS, 462, 751

García J. A., Kallman T. R., Bautista M., Mendoza C., Deprince J., Palmeri P., Quinet P., 2018a, in Mendoza C., Turck-Chiéze S., Colgan J., eds, ASP Conf. Ser. Vol. 515, The Problem of the High Iron Abundance in Accretion Disks around Black Holes. Astron. Soc. Pac., San Francisco, p. 282

García J. A., Steiner J. F., McClintock J. E., Remillard R. A., Grinberg V., Dauser T., 2015, ApJ, 813, 84

García J. A. et al., 2019, ApJ, 885, 48

García J. A. et al., 2018b, ApJ, 864, 25

García J. et al., 2014, ApJ, 782, 76

Jana A., Debnath D., Chakrabarti S. K., Mondal S., Molla A. A., 2016, ApJ, 107,819

Jiang J., Walton D. J., Fabian A. C., Parker M. L., 2019, MNRAS, 483, 2958

Kammoun E. S., Domcek V., Svoboda J., Dovciak M., Matt G., 2019, MNRAS, 485, 239

Kennea J. A. et al., 2011, Astron. Telegram, 3613, 1

Koyama K. et al., 2007, PASJ, 59, 23

Laor A., 1991, ApJ, 376, 90

Lohfink A. M., Reynolds C. S., Miller J. M., Brenneman L. W., Mushotzky R. F., Nowak M. A., Fabian A. C., 2012, ApJ, 758, 67
López K. M., Jonker P. G., Torres M. A. P., Heida M., Rau A., Steeghs D., 2019, MNRAS, 482, 2149

Martocchia A., Matt G., 1996, MNRAS, 282, L53

Matt G., Perola G. C., Piro L., 1991, A\&A, 247, 25

McClintock J. E., Shafee R., Narayan R., Remillard R. A., Davis S. W., Li L.-X., 2006, ApJ, 652, 518

Miller-Jones J. C. A., Sivakoff G. R., Rupen M., Altamirano D., 2011, Astron. Telegram, 3628, 1

Miller J. M., Reynolds C. S., Fabian A. C., Miniutti G., Gallo L. C., 2009, ApJ, 697, 900

Miller J. M. et al., 2013, ApJ, 775, L45

Miniutti G., Fabian A. C., 2004, MNRAS, 349, 1435

Mitsuda K. et al., 1984, PASJ, 36, 741

Nakahira S. et al., 2011, Astron. Telegram, 3626, 1

Negoro H. et al., 2011, Astron. Telegram, 3611, 1

Novikov I. D., Thorne K. S., 1973, in Dewitt C., Dewitt B. S., eds, Black Holes (Les Astres Occlus), Gordon and Breach, New York, p. 343

Rau A., Greiner J., Sudilovsky V., 2011, Astron. Telegram, 3619, 1

Reis R. C., Miller J. M., Reynolds M. T., Fabian A. C., Walton D. J., 2012, ApJ, 751, 34

Reynolds C. S., 2014, Space Sci. Rev., 183, 277

Reynolds C. S., Begelman M. C., 1997, ApJ, 488, 109

Ross R. R., Fabian A. C., 2007, MNRAS, 381, 1697

Russell T. D., Soria R., Miller-Jones J. C. A., Curran P. A., Markoff S., Russell D. M., Sivakoff G. R., 2014b, MNRAS, 439, 1390

Russell T. D., Soria R., Motch C., Pakull M. W., Torres M. A. P., Curran P. A., Jonker P. G., Miller-Jones J. C. A., 2014a, MNRAS, 439, 1381

Shakura N. I., Sunyaev R. A., 1973, A\&A, 24, 337

Strohmayer T. E., Smith E. A., 2011, Astron. Telegram, 3618, 1

Svoboda J., Dovčiak M., Goosmann R. W., Jethwa P., Karas V., Miniutti G., Guainazzi M., 2012, A\&A, 545, A106

Takahashi T. et al., 2007, PASJ, 59, 35

Tomsick J. A. et al., 2018, ApJ, 855, 3

Tripathi A., Nampalliwar S., Abdikamalov A. B., Ayzenberg D., Bambi C., Dauser T., García J. A., Marinucci A., 2019, ApJ, 875, 56

Verner D. A., Ferland G. J., Korista K. T., Yakovlev D. G., 1996, ApJ, 465, 487

Walton D. J., Nardini E., Fabian A. C., Gallo L. C., Reis R. C., 2013, MNRAS, 428, 2901

Walton D. J. et al., 2016, ApJ, 826, 87

Walton D. J. et al., 2019, MNRAS, 484, 2544

Wang Y., Ghasemi-Nodehi M., Guainazzi M., Bambi C., 2017, preprint (arXiv:1703.07182)

Wilkins D. R., Fabian A. C., 2012, MNRAS, 424, 1284

Wilkins D. R., Gallo L. C., 2015, MNRAS, 449, 129

Wilkins D. R. et al., 2012, MNRAS, 424, 217

Wilms J., Allen A., McCray R., 2000, ApJ, 542, 914

Xu Y. et al., 2018, ApJ, 852, L34

Yamada S. et al., 2012, PASJ, 64, 53

Young A. J., Ross R. R., Fabian A. C., 1999, MNRAS, 306, 461

Zdziarski A. A., Johnson W. N., Magdziarz P., 1996, MNRAS, 283, 193

Życki P. T., Done C., Smith D. A., 1999, MNRAS, 309, 561

This paper has been typeset from a $\mathrm{T}_{\mathrm{E}} \mathrm{X} / \mathrm{L} \mathrm{T} \mathrm{E} \mathrm{X}$ file prepared by the author. 